\title{
Chemins phonologiques
}

\author{
Jean - Pierre Montreuil \\ Université du Texas à Austin \\ ipmontreuil@,mail.utexas.edu
}

\section{Introduction}

Depuis plus de quinze ans, les postulats de la phonologie dérivationnelle (DT) ont été remis en question et l'attention des chercheurs s'est penchée sur le rôle des contraintes de surface. L'élaboration de modèles d'évaluation parallèle, notamment la théorie de l'optimalité (OT), a porté un nouvel éclairage sur l'analyse typologique des langues et sur l'apport de la théorie de la marque lorsqu'elle est encodée directement dans l'appareil grammatical. En contrepartie, un certain nombre de généralisations ont été perdues, notamment celles qui en DT se formulaient à un niveau de représentation intermédiaire. L'évaluation en parallèle perd la profondeur des analyses modulaires et séquentielles. Les efforts de récupérer cette profondeur n'ont rencontré qu'un succès mitigé. Les problèmes d'opacité demeurent.

De récents travaux (Jesney 2009, Kimper 2008, McCarthy 2006, 2007, 2008, Pruitt 2008, Wolff 2008) ont soutenu que les modèles d'OT qui utilisent des évaluations locales plutôt que globales aboutissent à des généralisations plus robustes en ce qui concerne la typologie et la variation phonologiques. En particulier, McCarthy (2007) a élaboré OT-CC, l'optimalité par les chaînes de candidats, une théorie de la fidélité susceptible de restorer certains éléments de cette dimension perdue.

L'objet du présent travail est d'évaluer cette approche au regard de quelques phénomènes de la phonologie du français. Pour maintenir une cohérence thématique, il s'agira uniquement de processus d'assimilation. Certains de ces processus - notamment ceux qui font référence au français standard - ont été décrits et parfois abondamment commentés, mais feront ici l'objet de réanalyses. D'autres - ceux qui font référence au français régional - n'ont pas, à notre connaissance, fait l'objet d'analyses antérieures.

Au-delà de l'aspect technique d'une telle entreprise, on discernera le souci majeur de retrouver un équilibre entre l'ancienne approche (DT), qui procédait "de haut en bas" i.e. vers l'output, qui disait ce qu'il fallait faire, mais sans dire pourquoi de façon explicite, et la nouvelle approche (OT), qui, elle, procède de bas en haut ie. à partir de l'output, qui dit ce qu'il ne faut pas faire, en expliquant pourquoi. La quête ultime reste définie: parvenir à une approche phonologique complète, qui donne le quoi (i.e. quel output est généré), le pourquoi (i.e. en vertu de quels principes grammaticaux, définis par l'encodage direct de la marque) et le comment (i.e. quel chemin relie l'input à l'output). C'est ce dernier élément qui s'impose comme le plus nouveau et c'est donc précisément le concept de chemin phonologique que ces pages cherchent à développer.

\section{Dominance et profondeur: la marque}

Pour permettre de comprendre comment la notion de dominance en OT et celle de profondeur en DT informent le concept de chemin phonologique, un exemple simple peut nous servir de point de départ.

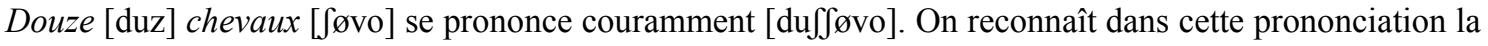
présence de deux processus assimilatoires tout à fait ordinaires: l'assimilation de voix et l'assimilation de hauteur.

Dans le format traditionnel de DT, utilisant une règle linéaire de transformation, l'assimilation de voix entre deux obstruantes prend la forme suivante ${ }^{1}$ :

(1) Assim-Vx: [-son] $\rightarrow[\alpha v x] /[$ [-son, $\alpha v x]$

(les obstruantes s'assimilent en voix aux obstruantes qui les suivent) 


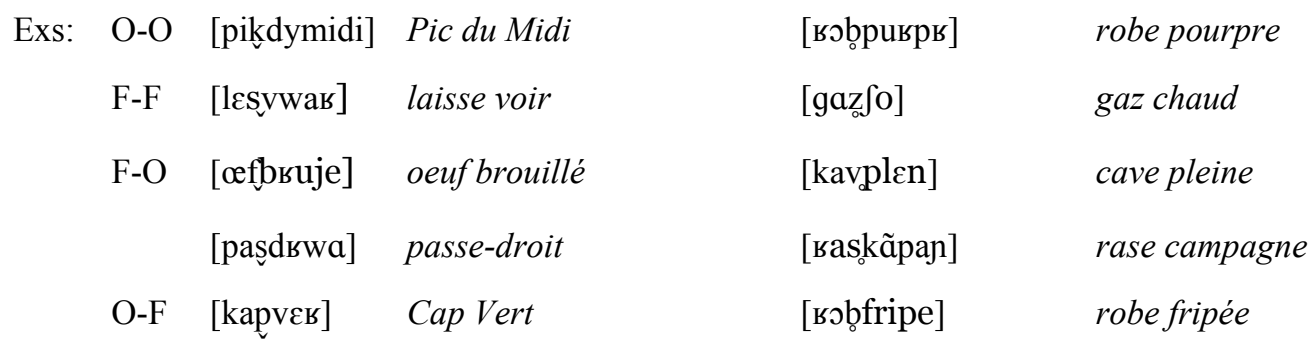

L'assimilation de hauteur, par contre, n'affecte que les fricatives coronales:

(2) Assim-Ht: $[+$ cons, + cont, + cor $] \rightarrow[+$ haut $] /$ [+cons, + cont, + cor, +haut $]$

(les fricatives coronales s'assimilent en hauteur aux fricatives coronales hautes qui les suivent)

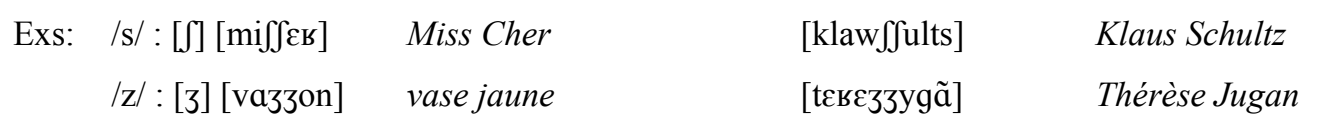

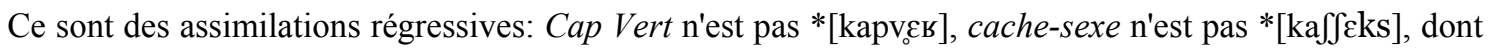
le comportement ne pose aucun problème et qui ont été communément relevées. Mais plus que les mécanismes eux-mêmes, déjà décrits et analysés de multiples perspectives (Duez 1995, Jun 1995, Nolan 1992, pour le français, mais aussi plus généralement Ernestus 2000, Gow 2000, Gow 2004, Kuzla 2003, Nolan et al 1996, Wetzels \& Mascaro 2001), c'est l'interaction de ces deux processus qui retient ici notre attention. Les exemples suivants cumulent les deux processus:

$$
\begin{array}{ll}
/ \mathrm{s} /:[3] & {[\mathrm{kaz3} \tilde{\mathrm{a}}]} \\
/ \mathrm{z} /:\left[\int\right] & {\left[\mathrm{ga} \iint \mathrm{o}\right]}
\end{array}
$$

casse jambes

gaz chaud

$$
\text { [mizзiьõd] }
$$

Miss Gironde

Charcot

On notera donc qu'une telle grammaire fusionne en [duzzymã], au prix d'une certaine ambiguïté, les prononciations de douces juments et douze juments. Des études sur l'accès lexical et la perception des suites assimilées ont montré l'importance des indices secondaires, notamment des variations de durée vocalique et de transitions acoustiques (Hallé et al 2000, Quené et al 1998, Snoeren 2005, Snoeren et al 2006, Snoeren et al 2008, Weber 2001, Weber 2002).

Dans la perspective qui nous préoccupe, l'intérêt vient du fait que, pour s'intégrer dans une grammaire cohérente, qu'elle soit formulée en OT ou en DT, ces deux assimilations doivent trouver leur place, et cette place est déterminée par leur nature. En effet, l'assimilation de hauteur est un processus semiautomatique qui n'affecte qu'une classe très restreinte de sons, qui prend couramment une dimension stylistique et dont les lecteurs sont souvent conscients. Son absence est ressentie comme reflétant une

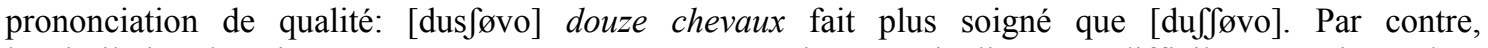
l'assimilation de voix est un processus purement automatique, particulièrement difficile à supprimer, dont les locuteurs sont rarement conscients (en fait, ils en nient fréquemment l'existence). Il est aussi plus courant dans les langues du monde. En français, son absence est ressentie comme reflétant une prononciation non-naturelle: [duz Øøvo] et [duzโøvo] font moins naturel que [dus Øøvo].

Les postulats de la théorie dérivationelle veulent que les processus purement phonétiques (automatiques, sans exception, sans dimension stylistique etc...) soient les plus superficiels et localisés dans les derniers stades de la dérivation (on les qualifie de tardifs ou "late rules" en anglais). C'est même probablement la qualité principale de DT que d'avoir su construire et formaliser une théorie de l'évolution des processus, en dévoilant comment les processus nouvellement créés s'intègrent à la grammaire par le bas de la dérivation, puis au fur et à mesure que d'autres processus viennent à leur tour s'incorporer à la langue, les premiers se phonologisent, s'opacifient, perdent leur justification phonétique, se morphologisent, s'entachent d'exceptions, voire se lexicalisent et disparaissent. Dans l'exemple simple de /zJ/: [J]], l'ordre suivant s'imposera: 
En optimalité, les évaluations s'opèrent de façon parallèle, et les réinterprétations de l'input correspondent de façon usuelle à la dominance d'une contrainte de marque, qui optimisera l'output en une forme moins marquée, et prendra le pas sur une contrainte de fidélité qui cherche à maintenir inchangé la forme de l'input. Ce rapport de force innovation vs. conservation a été longuement exploré depuis le début des années 1990. Dans l'exemple que nous avons retenu, l'assimilation de hauteur est exprimée par l'ordre des contraintes * CONT-Ht $\gg$ IDENT-Ht, signifiant qu'il est plus important en style naturel d'éviter un contour de hauteur que de garder inchangée la hauteur des consonnes. A Assim-Vx correspond l'ordre *CONT-Vx $\gg$ IDENT-Vx, signifiant qu'il est plus important d'éviter un contour de voix que de garder inchangée la voix de l'input. On ajoutera seulement une contrainte de fidélité positionnelle IDENT-Att pour refléter la directionalité (régressivité) de l'assimilation et bloquer *[duzzøvo]. Les contraintes peuvent se paraphraser comme suit ${ }^{3}$ :

$$
\begin{array}{ll}
{ }_{\text {CONT-Ht }} & =\text { pas de contour de hauteur entre obstruantes coronales } \\
\text { IDENT-Ht } & =\text { la hauteur d'une consonne en input reste inchangée en output } \\
{ }^{*} \text { CONT-Vx } & =\text { pas de contour de voix entre obstruantes } \\
\text { IDENT-Vx } & =\text { la voix d'une consonne en input reste inchangée en output } \\
\text { IDENT-Att } & =\text { les traits des consonnes d'attaque restent inchangés }
\end{array}
$$

\begin{tabular}{|c|c|c|c|c|c|c|}
\hline & $/ \mathrm{z} \mathrm{g} /$ & IDENT-Att & ${ }^{*}$ CONT-VX & ${ }^{*}$ CONT-Ht & IDENT-Vx & IDENT-Ht \\
\hline $\mathrm{a}$ & {$\left[\mathrm{z} \int\right]$} & & $* !$ & * & & \\
\hline $\mathrm{b}$ & {$\left[\mathrm{s} \int\right]$} & & & $* !$ & * & \\
\hline c & $[3]]$ & & $* !$ & & & $*$ \\
\hline d & {$\left[\iint\right]$} & & & & $*$ & $*$ \\
\hline e & {$[\mathrm{zz}]$} & $* !$ & & & * & * \\
\hline
\end{tabular}

Ainsi, le tableau qui suit sélectionnerait /zJ/ : [S] comme optimal.

Tableau 1. Douze chevaux

Revenons un instant sur la façon dont s'articule le lien logique entre ces deux approches. Un certain nombre d'idées spécifiques ont été exploitées dans le cadre de la phonologie lexicale dérivationnelle, qui visaient toutes à établir une corrélation entre la place d'un processus dans la grammaire et sa fonction dans la langue. Par exemple, Kiparsky (1985), Mohanan (1986) dressent une liste des propriétés des processus lexicaux s'appliquant tôt dans (= en haut de) la dérivation, y compris leur fréquente opacité, récursivité, binarité, leur conditionnement morphologique et leur souci de préserver les structures extantes. Les processus post-lexicaux s'appliquant tard dans (=en bas de) la dérivation offrent les propriétés contraires: ils peuvent affecter le mot dans la phrase, ils sont graduels, non-itératifs, transparents et ne souffrent pas d'exceptions. Le locuteur n'en est pas conscient, ils ne sont pas conditionnés par la morphologie ou le lexique, et ils créent librement des sons nouveaux en allophonie.

Le concept plus général voudrait que plus le processus est placé haut dans la grammaire, plus il est contrôlable et suppressible. Ce concept reste valide en dehors de tout formalisme opposant des modules lexicaux et post-lexicaux. Le haut d'une dérivation se perd dans l'arbitraire du signe tandis que le bas a subi toutes les régularisations de la grammaire et rejoint le domaine des réflexes et de l'ancrage biologique. En OT, un processus difficilement contrôlable et suppressible correspond à l'expression d'une 
contrainte forte, i.e. que l'on s'attend à trouver en position dominante. Il n'est donc pas surprenant que, dans le tableau $1,{ }^{*}$ CONT-VX domine *CONT-HT.

Si le travail d'évaluation d'un tableau se bornait à sélectionner le candidat optimal, ce rapport entre la dominance (en OT) et la profondeur (en DT) ne se trouverait pas exprimé. Dans le tableau 1, le candidat d. est optimal quel que soit l'ordre des contraintes de marque. Mais s'il s'avère, comme nous l'avons noté, que [duzðøvo], candidat c., est moins naturel que [dus øøvo], candidat b., l'ordre présenté est le seul ordre logique. En dehors des intuitions individuelles, s'il s'avère que l'assimilation de hauteur est facilement contrôlable tandis que l'assimilation de voix est difficilement suppressible, l'ordre présenté est encore l'ordre logique. C'est donc par sa capacité d'aller au-delà de la sélection du candidat optimal et de représenter des degrés de désirabilité, autrement dit la relativité de la marque, que l'évaluation en parallèle peut elle aussi refléter une certaine profondeur.

On pourrait alors chercher à mener cet argument jusqu'à son point le plus fort et établir l'équation suivante entre les deux analyses: "Si un processus haut placé dans la dérivation en DT est reflété en OT par un ordre de contrainte M1 $\gg$ F1 et qu'il est suivi d'un second processus moins haut placé dans la dérivation reflété par un ordre de contraintes M2 $\gg$ F2, l'ordre M2 $\gg$ M1 s'impose." En d'autres mots, la contrainte de marque responsable du processus le plus superficiel et automatique dominera toujours celle qui engendre le processus profond.

Si la position est forte, l'argument reste à prouver. Mais il est légitime de continuer de penser que si la grammaire doit impérativement refléter le fait que deux processus d'assimilation jouent un rôle différent et occupent une place différente, c'est la théorie dérivationnelle qui, des deux théories, formalise le plus clairement cet impératif. Dans la section suivante, on se posera une question différente, mais parallèle: si la relativité de la marque ne représente que partiellement la profondeur d'une dérivation, qu'en est-il de la fidélité ?

\section{Les écarts de fidélité}

Dans une série de travaux dont le but est de proposer une solution globale aux problèmes d'opacité en optimalité, McCarthy $(2006,2007,2008)$ échafaude un modèle d'évaluation de candidats en chaîne (OT$\mathrm{CC}$ ). Dans le modèle classique en OT, la grammaire évalue une infinité de formes phonémiques ou phonétiques (leur statut n'est pas toujours clair), parfois enrichies d'information structurale. Dans le modèle OT-CC, ces formes deviennent les points terminaux de chaînes. Parce que ces chaînes doivent obéir à des conditions de bonne formation, le nombre de candidats à évaluer n'est plus infini. Seuls des candidats générés par des chaînes valides seront retenus.

Une chaîne est constituée de maillons et chaque maillon d'une chaîne diffère du maillon précédent par un écart de fidélité (EF; en anglais, 'localized unfaithful mapping', ou LUM). Par exemple, dans la région picarde où l'on observe la même assimilation de hauteur consonantique qu'en français, il existe des zones où cette assimilation s'accompagne d'une dégémination --on dirait en DT que l'assimilation précède et alimente la dégémination--, douze juments se prononce [duzymã] et la chaîne suivante s'imposera: < duzzymã, duz̧ymã, duzymã $>$. Or, en OT-CC, l'évaluation grammaticale prend une double forme: ce qui est évalué est à la fois la forme terminale, i.e. la marque de l'output, et les écarts de fidélité que commettent les différents maillons de la chaîne. C'est ce dernier point qui contitue pour OT l'élément nouveau et c'est cette innovation qui fait que OT-CC est avant tout une théorie de la fidélité en OT. McCarthy l'énonce clairement (2007:4):

The evaluation of a candidate chain by the grammar has some familiar properties: markedness constraints assess the last form in the chain, which is the chain's output; and faithfulness constraints measure discrepancies between the first and last forms in the chain.

Pour être bien formées, les chaînes doivent obéir à des critères d'harmonicité et de gradience. Ainsi, chaque maillon doit s'écarter du précédent de la façon la plus harmonique possible, au vu de la grammaire

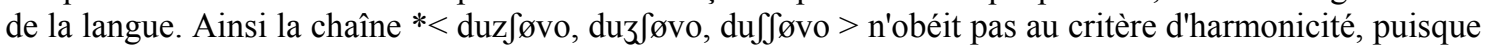


le premier EF n'est pas le plus harmonique (revoir tableau 1); c'est au contraire la chaîne $<$ duzføvo,

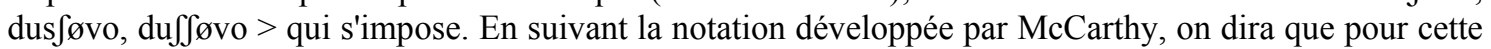
dernière chaîne, la séquence d'EF est la suivante:

$$
\begin{aligned}
& \text { chaîne séquence d'EF }
\end{aligned}
$$

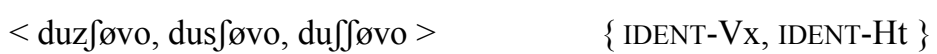

De plus, la gradience joue un rôle important. Chaque maillon ne doit différer du précédent que par un seul

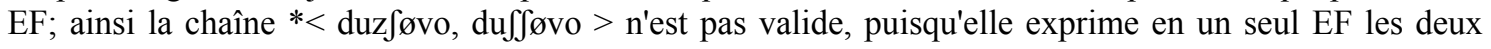
assimilations, de voix et de hauteur.

Il reste important de souligner, comme le fait McCarthy (2007) à plusieurs reprises, que chaînes de candidats et dérivations ne doivent pas être confondues. Ces dernières n'ont jamais eu l'ambition de faire procéder une forme phonologique vers des alternatives de moins en moins marquées. Dans le formalisme DT, des règles arbitraires et même phonétiquement peu plausibles (les "crazy rules" des années 1960-70) peuvent fort bien trouver leur place à n'importe quel stade d'une dérivation. Par contre, et en dépit des critiques que l'on a pu formuler contre l'idée même que les contraintes optimisent systématiquement les formes phonologiques, OT-CC maintient l'objectif d'optimisation, même si celui-ci se recherche avant tout au niveau local, comme l'explique McCarthy (2007: 93):

\begin{abstract}
The harmonic improvement requirement on candidate chains, when combined with a specific theory of LUMs and faithfulness, makes restrictive predictions about which phonological mappings are and are not possible. Essentially, OT-CC requires that complex mappings show local improvement on the way to global improvement. Classic OT, on the other hand, permits complex mappings that produce global harmonic improvement but are not decomposable into any chain of local improvements.
\end{abstract}

C'est là une distinction essentielle qui impacte à la fois le concept de chemin phonologique et la direction qu'un chemin est susceptible de prendre. Ceci reconnu, il n'en reste pas moins vrai que dans leur procédure, DT et OT-CC présentent de nombreux points communs et McCarthy n'hésite pas à caractériser à plusieurs reprises OT-CC comme une synthèse entre OT et DT.

Pour résumer cette section, nous dirons que nous nous dirigeons vers une position selon laquelle la grammaire se doit d'évaluer à la fois un point d'arrivée, et c'est surtout là le rôle de la marque, et un chemin qui mène à ce point d'arrivée, et c'est surtout là le rôle de la fidélité. L'élaboration de chaînes phonologiques, telle qu'elle se pratique en OT-CC, permet de formaliser ce cheminement. Dire que les

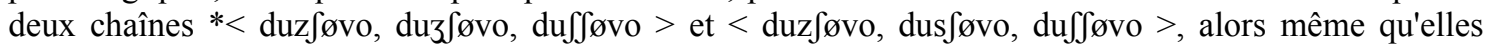
mènent du même point de départ au même point terminal, sont en compétition et que c'est la seconde qui est retenue par la grammaire, permet une richesse d'analyse et de compréhension que DT ou OT à elles seules ne peuvent pas atteindre.

\title{
4 Chemins parallèles, chemins obligatoires
}

Existe-t-il des chemins parallèles, des chemins obligatoires ? Considérons la façon dont l'assimilation de voix entre en interaction avec une autre assimilation, celle de la nasalité.

En style relâché, le français connaît un processus d'assimilation progressive de nasalité; on entend

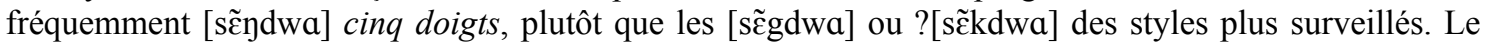
phénomène est bien connu: lorsqu'elle suit une voyelle nasale, une plosive se réalise en nasale sans perdre son point d'articulation.

$$
\begin{aligned}
& {[\mathrm{p}],[\mathrm{b}]>[\mathrm{m}][\text { lãmdøfve] lampe de chevet [3ãmdøbwa] jambe de bois }} \\
& {[\mathrm{t}],[\mathrm{d}]>[\mathrm{n}] \text { [vẽndø] vingt-deux [lãndølese] lande de Lessay }} \\
& {[\mathrm{k}],[\mathrm{g}]>[\mathrm{\eta}] \text { [bãydøfrãs] Banque de France [lõyduf] longue douche }}
\end{aligned}
$$


Le mécanisme d'assimilation s'exprime en phonologie autosegmentale par 1) la réassociation du noeud NASAL de la voyelle ('spreading') et 2) la désassociation du noeud NASAL de la consonne suivante ('delinking'). Le contexte est sujet à variation. Dans le français du Canada, on entend ces plosives assimilées même en fin de phrase (Walker 1984):

(6) J'ai mal aux jambes [3ãm]

Ça fait beaucoup de monde [mõn]

C'est une belle langue [lãy]

Le français de France requiert en général que la plosive assimilée soit suivie d'une consonne. Dans le parler de l'auteur (appelons-le Parler 1, de type conservateur), cette consonne doit être une obstruante sonore et on entend l'assimilation dans Banque de France mais pas dans Banque Populaire. D'autres variantes (disons Parler 2) sont moins restrictives et assimilent même devant les obstruantes sourdes (7) tandis que d'autres, plus tolérantes encore, connaissent le phénomène même devant une sonante, comme par exemple la liquide [1] en (8):

$$
\begin{aligned}
& \text { ? [зãmotutruz] } \\
& \text { ? [sẽ̄otwa] } \\
& \text { ? [1ãmเวьك] }
\end{aligned}
$$

$$
\begin{array}{lll}
\text { une [3ãm] de pantalon } & \text { et } & \text { ?? tu as les [3ãm] / [3ãa] lourdes } \\
\text { le [mõn] du silence } & \text { et } & \text { ?? c'est un [mõn] / [mõd] libre } \\
\text { la [lãy] de bois } & \text { et } & \text { ?? la [lãg] / [lãg] latine }
\end{array}
$$

La caractérisation des Parlers 1 et 2 en OT-CC nous informe sur la nature des chemins phonologiques. L'assimilation de nasalité relève de la dominance de la marque, encore une fois une contrainte de contour, sur la fidélité à l'oralité consonantale: *CONT-ṼO »IDENT-[-nas].

$$
\begin{array}{ll}
{ }^{*} \text { CONT- } \mathrm{V} O & =\text { pas de contour voyelle nasale }+ \text { consonne orale devant consonne } \\
\text { IDENT-[-nas] } & =\text { l'oralité d'une consonne en input reste inchangée en output }
\end{array}
$$

Pour ce qui est de la suite des écarts de fidélité dans l'interaction entre voix et nasalité, il faut reconnaître une suite \{ IDENT-Vx, IDENT-Ht $\}$ dans le parler 1. En effet, si /k/> [n] (ou plutôt [n] ) ne s'entend pas devant consonne sourde, comme dans Banque Populaire, c'est que l'assimilation de voix est une condition nécessaire à l'assimilation de nasalité. On a donc un chemin obligatoire: $\mathrm{k}>\mathrm{g}>\mathrm{y}$

chaîne

$$
\text { < bãkdøfrãs, bãgdøfrãs, bãydøfrãs > }
$$

séquence d'EF

\{ IDENT-Vx, ident-[-nas] \}

Par contre, on peut concevoir qu'une phonologie qui accepte [bãypopyles] - c'est le Parler 2 -, avec k $>\eta_{0}$ et assimilation de voix non-applicable, prenne deux chemins différents pour Banque de France: $\mathrm{k}>\mathrm{g}>\mathrm{y}$ ou bien $\mathrm{k}>\mathrm{\eta}_{\mathrm{o}}>\mathrm{\eta}$.

Dans de plus longs "mappings" input-output, tels que
(9) I: / /ãbrdami/
O: [Jãmdami]
I: /pẽttrdydimã $/ /$
O: [pẽndydimãf]
I: /kõgвdøвоље/
O: [kõydøво $\mathrm{e}]$
I: /عgzãpldøprekosite/
O: [عgzãmdøprekosite]

\begin{abstract}
chambre d'amis
peintre du dimanche

congre de rocher

exemple de précocité
\end{abstract}


une chaîne plus précise s'établit qui prend en compte le voisement consonantique, la simplification des codas et la nasalisation progressive des obstruantes. Si encore une fois on ignore la possibilité d'un schwa final dans le premier mot - dans un souci de simplification, alors même que sa présence serait tout à fait aussi logique que dans la préposition de -, on dira que la suite de consonnes /OL\#d/ est "réparée" ou par l'épenthèse vocalique (on aura alors [Egzãplødø]) ou par la simplification des codas (chute du [1] violant MAX-[liq]). Dans ce dernier cas, l'assimilation de nasalité ne peut se produire qu'après la chute des liquides: *[Egzãml] n'est pas une option. Le voisement et la nasalisation de la consonne n'ont donc de sens que si elles font suite à la simplification du groupe $/ \mathrm{pl} /$. Ce cheminement s'accorde avec la nature du processus de simplification, phénomène contrôlable, dont la dimension stylistique et même lexicale (?? la Bib' de Gutenberg) n'est pas contestée.

(10) Input:

Simplification

Assimilation de voix

Assimilation de nasalité

Output:

chaîne

<..ãpldø.., ..ãpdø.., ..ãbdø.., ..ãmdø..>

\author{
/عgzãpldøprekosite/ \\ /.......ãpdø................ \\ /.......äbdø................/ \\ |.......ãmdø................ \\ [عgzãmdøprekosite] exemple de précocité \\ séquence d'EF \\ \{ MAX-[liq], IDENT-[vx], IDENT-[-nas] \}
}

\section{Chemins et points d'arrivée}

Nous avons développé plus haut l'idée centrale de cet article, à savoir que l'analyse phonologique ne doit pas se contenter, comme le fait DT, de déterminer des tracés vers des formes terminales sans les baser sur des considérations de marque incorporées à la grammaire. Elle ne peut pas non plus se contenter, comme le fait OT, de justifier la désirabilité d'un point d'arrivée, une forme d'output. Il faut à la fois élaborer l'optimalité du chemin et celle du but.

Il existe en phonologie de nombreux types d'interaction qui pourraient illustrer comment ces deux objectifs se distinguent. Cette section en choisit deux: l'un constitue un chemin opaque, connu sous le nom anglais de "counterbleeding" ou appauvrissement virtuel, qui sera ici illustré à partir d'un exemple régional. L'autre, connu sous le nom anglais de NDEB, Non-Derived Environment Blocking, ou blocage en environnement non-dérivé (je garderai l'acronyme anglais), sera examiné à partir d'un phénomène très connu du français standard.

L'opacité phonologique se manifeste lorsque l'interaction de deux ou plusieurs processus génère une forme à partir de laquelle l'existence ou la raison d'être du premier des deux processus n'est plus percevable. Par exemple, si une allophonie se crée à partir d'une source mais que cette source se trouve oblitérée plus tard dans la dérivation, l'allophonie n'est plus compréhensible. De nombreux cas ont été relevés où il est clair que chacun des processus en isolation est transparent et productif et que c'est uniquement lorsqu'ils entrent en interaction que l'opacité se manifeste.

Tous les modèles d'analyse qui sont basés sur des évaluations d'output - ce que l'anglais appelle "outputdriven" models - se trouvent donc confrontés à l'opacité. Par contre, pour les théories qui, comme DT, peuvent formuler des généralisations à des niveaux intermédiaires, l'opacité ne constitue pas un problème majeur. Une myriade de propositions ont été suggérées pour équiper OT des outils nécessaires au traitement de l'opacité, propositions dont la plupart étaient porteuses de nouveaux problèmes, et il est légitime d'affirmer qu'aucune d'entre elles n'a su ou même prétendu apporter une solution globale à ce dilemme.

Dans le cadre d'OT-CC, McCarthy suggère d'utiliser des contraintes de Précédence, qui ont pour objet de favoriser certaines relations de précédence parmi les constituants d'une chaîne d'EF et d'en pénaliser 
d'autres. Le concept de précédence ne fait pas référence à l'ordre des contraintes dans les tableaux, mais uniquement à celui des écarts de fidélité dans la séquence extraite d'une chaîne. Ainsi, dans l'interaction de deux contraintes $\mathrm{A}$ et $\mathrm{B}$, une contrainte de précédence $\operatorname{PREC}(\mathrm{A}, \mathrm{B})$ exigera que tout écart de fidélité qui viole $\mathrm{B}$ soit précédé et non-suivi dans la même chaîne d'un EF qui viole A. Par exemple, dans le cas de figure cité plus haut, une contrainte de précédence pourra pénaliser toute chaîne où l'effacement de la source n'est pas précédé de la création de l'allophonie.

Le bas-normand du Cotentin connaît une telle situation, dans son dialecte, mais occasionnellement aussi dans son français régional (Montreuil 1992). On observe dans ce parler une assimilation progressive de $[\mathrm{r}]$ après consonne, lorsqu'un schwa intervient. Ce phénomène est donc remarquable pour deux raisons distinctes: la directionalité de l'assimilation (l'assimilation non-persévérative existe aussi mais elle est beaucoup plus rare), et la non-adjacence en input des source et cible de l'assimilation. De plus, la source varie sous-dialectement: dans certaines régions, l'assimilation est limitée aux coronales; dans d'autres, elle se produit après toutes les consonnes. Il existe un continuum entre les parlers non-assimilants et les parlers les plus assimilants:

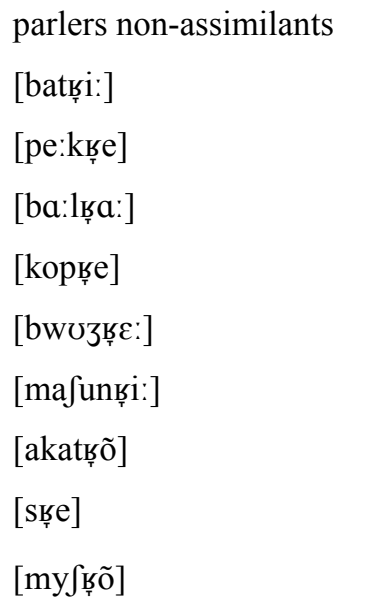

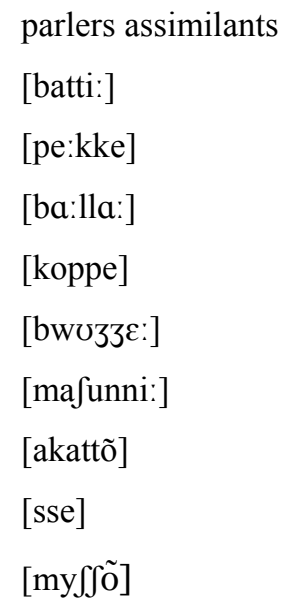

batterie
pêcherai
donneras (bâilleras)
couperai
bougeraient
maçonnerie
achèterons
serai
mucherons (= cacherons)

Il s'agit bien ici de suites dérivées: quelle que soit la représentation du schwa que l'on choisit de retenir (les avis sont très partagés sur la question), les inputs sont ici de type $\mathrm{C}+$ schwa $+[\mathrm{b}]$, avec des alternances communes du type [lavvõ] laverons vs. [laveвjõ] laverions (le e muet régional se réalise en [e]). De façon cruciale, l'assimilation ne se fait pas dans des suites [ $\mathrm{Cr}]$ non-dérivées. On dit patrie [patкi:] et non *[patti:], pendrais [pãdre] et non *[pãdde], avril [avвi] et non *[avvi].

La raison phonétique de cette divergence est à trouver dans la nature faible du ' $r$ ' intervocalique dans ces parlers. On le constate dans de nombreuses formes dialectales où il se montre ou affaibli (ex: [t $\mathrm{f} y$ he] pour curé), ou absent ([t]ye]). La faiblesse de l'approximante [ธ̌] fait qu'elle se prête à l'assimilation, une fois l'adjacence mise en place. Le [в] fricatif de patrie, lui, ne s'assimilera pas. Dans cette perspective, la dérivation s'établit comme suit ${ }^{4}$ :

/batəьі:/ : [batti:] en DT

$$
\text { /batəьi:/ }
$$

batterie

Lénition intervocalique /s/

$$
/ \mathrm{B} / \rightarrow[\mathrm{b}] / \mathrm{V}
$$

Mise en adjacence surapplication

$$
[ə] \rightarrow \varnothing / \mathrm{VC}
$$
parlers non-assimilants

Assimilation des approximantes 


$$
[+ \text { approx }] \rightarrow[\alpha \mathrm{T}] /[\alpha \mathrm{T}]
$$

(13) /batәьі:/: [batti:] en OT

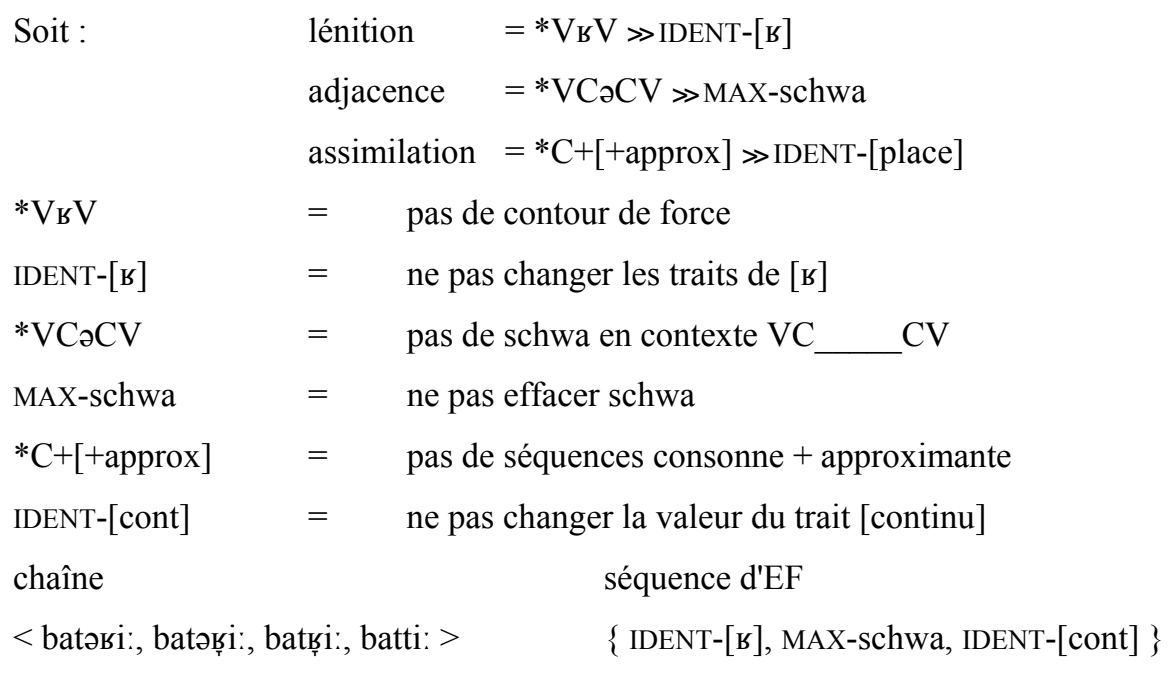

Aucune contrainte ne motive l'assimilation du [в] fort dans patrie, qui reste inchangé. Toute autre chaîne reliant I: / batəsi: / O: [batti:] serait incomplète ou défectueuse. L'opacité est engendrée dès le début de la chaîne: la mise en adjacence détruit l'environnement nécessaire au processus de lénition, qui se trouve donc en surapplication. Il faut donc, avant même de parler de l'assimilation, établir la grammaire des parlers non-assimilants et expliquer pourquoi le candidat opaque [bațָi:] l'emporte sur un candidat transparent [batri:]. Le tableau 3 est composé des quatre contraintes suffisant à exprimer et la lénition et l'adjacence, mais il est défectueux puisque c'est d. et non c. qui l'emporte. Il présente un ordre plausible, avec la marque dominant la fidélité. Evidemment, d'autres ordres sont possibles, mais on ne s'attardera pas à les explorer puisqu'il apparaît dès l'abord qu'aucun de ces ordres ne rétablirait c. comme optimal (d. ne viole qu'un sous-ensemble des contraintes que viole c.). C'est le schéma typique de la surapplication.

\begin{tabular}{|c|c|c|c|c|}
\hline / batәьі: / batterie & *VиV & $* \mathrm{VC} \curvearrowright \mathrm{CV}$ & MAX-schwa & ident-[ъ] \\
\hline а. [bаtәкі:] & $* !$ & * & & \\
\hline b. [batəôi:] & & $* !$ & & * \\
\hline opaque c. [batw̦i:] & & & $*$ & $* !$ \\
\hline transparent d. [batкi:] & & & * & \\
\hline
\end{tabular}

Tableau 2. Parlers non-assimilants: tableau défectueux (c. perd)

La séquence d'EF de c. est \{ IDENT-[в], MAX-schwa \}, celle de d. est simplement \{ IDENT-[в] \}: la contrainte de précédence qui pénalise $d$. pour avoir créé l'adjacence sans avoir affaibli $[\mathrm{r}]$ au préalable, rétablira la justesse du tableau. Nous la dénommons simplement PREC dans les tableaux 3 et 4 , et comme sa paraphrase ci-dessous l'indique, elle se trouve enfreinte lorsque, dans la séquence d'écarts de fidélité extraite de la chaîne d'un candidat, un écart spécifique n'est pas précédé d'un autre écart qui lui donne son sens. Ici, un candidat qui aurait négligé de prendre le chemin: "lénition, puis adjacence", se voit pénalisé par la contrainte d'adjacence; c'est comme si, pour exploiter à nouveau notre métaphore, il s'était fourvoyé.

Séquence d'EF: < IDENT-[r], MAX-schwa >

Précédence: PREC(IDENT-[в], MAX-schwa) = EF IDENT-[в] doit précéder EF MAX-schwa 


\begin{tabular}{|l|c|l|c|c|c|}
\hline /batəвi: / & $* \mathrm{~V}[\mathrm{в}] \mathrm{V}$ & $* \mathrm{VCəCV}$ & MAX-schwa & PREC & IDENT-[в] \\
\hline c. [bații:] & & & $*$ & & $*$ \\
\hline d. [batкi:] & & & $*$ & $* !$ & \\
\hline
\end{tabular}

Tableau 3. Précédence de l'EF IDENT-[в] (с. gagne)

L'adjacence alimente l'assimilation dans les parlers assimilants. Le tableau 4 présente la grammaire complète de la relation / batəsi: / : [batti:]. $\mathrm{Si} * \mathrm{C}+[+\mathrm{appr}$ ] s'insère juste devant IDENT-[в], le candidat $\mathrm{c}$. (la forme lénifiée) reste la meilleure alternative.

\begin{tabular}{|c|c|c|c|c|c|c|c|}
\hline /batəsi: / batterie & *VвV & *VCəCV & MAX-schwa & $\begin{array}{c}\text { PRE } \\
\text { C }\end{array}$ & ${ }^{*} \mathrm{C}+[+$ appr $]$ & IDENT -[B] & IDENT-[cont] \\
\hline а. [batәві:] & $* !$ & * & & & & & \\
\hline b. [batəถ̦i:] & & $* !$ & & & & * & \\
\hline c. [bațָi:] & & & $*$ & & $* !$ & $*$ & \\
\hline d. [bаtкі:] & & & $*$ & $* !$ & & & \\
\hline e. [batti:] & & & & & & & * \\
\hline
\end{tabular}

Tableau 4. Parlers assimilants: /batәві:/ : [batti:]

Dans la prononciation régionale de notre exemple douze chevaux telle qu'elle est rapportée par Carton et al (1983) pour le français de Picardie, il apparaît qu'une interaction plus précise s'établit. Le français de Picardie tend à simplifier les suites de consonnes identiques, par dégémination: $\mathrm{Ci} \mathrm{Ci} \rightarrow \mathrm{Ci}$, et à prononcer [duføvo] douze chevaux et [duzymã] douze juments. A l'ambigüité du français douces juments : douze juments, le picard ajoute donc celle de doux chevaux : douze chevaux. Les formes dégéminées du picard représentent un cas d'opacité, au sens où l'input / duz øøvo / n'est pas récupérable à partir de [du〕øvo]; la dégémination masque les processus d'assimilation. En picard, il faut que la chaîne $<$ duz øøvo,

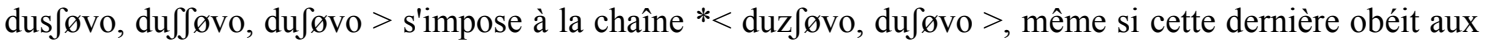
critères de gradience (un seul écart de fidélité) et d'harmonicité. Là aussi, la précédence devra intervenir, pour imposer le chemin 'assimilation puis dégémination'.

Reprenons le détail de ces processus, à partir de douze juments pour n'avoir à considérer qu'une seule assimilation. Il est clair que d'une manière générale, l'effacement des codas - et plus encore celui des attaques - est fortement pénalisable. C'est donc uniquement l'interaction avec l'effacement d'une partie de géminée que nous considèrerons ici, et nous le pénaliserons par MAX-Ci pour simplifier. Quant à la dégémination, qui fait préférer [3] à [33] dans douze juments, elle indique qu'une troisième contrainte de contour, formulée au niveau du noeud-racine ('root-node'), domine à son tour MAX-Ci (il est préférable d'éviter une géminée que la chute d'une consonne). Il s'agit d'un effet de type OCP (Obligatory Contour Principle).

$\begin{array}{lll}\mathrm{OCP} & = & \text { pas de géminée (contour de traits obligatoire) } \\ \mathrm{MAX}-\mathrm{Ci} & = & \text { pas d'effacement de consonne en structure géminée } \\ \mathrm{PREC}(\text { IDENT-Ht, MAX-Ci) } & = & \text { l'EF d' assimilation doit précéder l'EF de dégémination }\end{array}$

(14) douze juments (dégémination en picard)

chaîne

séquence d'EF 


$$
<. .23 . ., . .33 . ., . .3 . .>
$$

\{ IDENT-[ht], MAX-Ci \}

L'analyse est la même que dans le cas du bas-normand cotentinois si l'on considère que la dégémination correspond à un processus $\mathrm{C}_{1} \mathrm{C}_{2}>\mathrm{C}_{1}$ ie. dans la suite parlée, la deuxième consonne ne devient plus nécessaire une fois le contexte créé par la première. Il y a alors surapplication dans la dernière étape de l'évolution [duzzymã $]>\left[\right.$ duz $\left.{ }_{1}{ }_{2} y m a \tilde{a}\right]>[$ dużymã $]$.

\begin{tabular}{|l|c|c|c|c|}
\hline$/$ z 3/ douze juments & $*$ CONT-Ht & OCP & MAX-Ci & IDENT-Ht \\
\hline a. $[\mathrm{z}$ 3] & $* !$ & $*$ & & \\
\hline b. $[3$ 3] & & $* !$ & $*$ & $* !$ \\
\hline opaque c. $[31]$ & & & $*$ & \\
\hline transparent d. $\left[3_{2}\right]$ & & & $*$ & \\
\hline
\end{tabular}

Tableau 5. Douze juments en picard: tableau défectueux (c. perd)

Pour les candidats optimaux, la séquence d'écarts de fidélité suit le chemin $<$ IDENT-Ht, MAX-Ci $>$ pour douze juments et < IDENT-Vx, IDENT-Ht, MAX-Ci > pour douze chevaux. Les candidats transparents obtiennent une non-géminée par force brutale (chute de consonne non-assimilée); cette procédure n'est pas justifiée par la grammaire. La contrainte de précédence PREC(IDENT-Ht, MAX-Ci) rétablit le candidat opaque comme optimal en pénalisant le candidat transparent pour ne pas avoir assimilé avant de dégéminer.

\begin{tabular}{|l|c|c|c|c|c|}
\hline /z 3/ douze juments & $*$ CONT-Ht & OCP & MAX-Ci & PREC(IDENT-Ht, MAX-Ci) & IDENT-Ht \\
\hline opaque c. $\left[3_{1}\right]$ & & & $*$ & & $*$ \\
\hline transparent d. $[32]$ & & & $*$ & $* !$ & \\
\hline
\end{tabular}

Tableau 6. Précédence de l'EF IDENT-Ht (c. gagne)

La semi-vocalisation en français standard peut se concevoir sous la perspective du blocage de formes lexicales (NDEB), mentionné plus haut. On parle explicitement de NDEB lorsqu'un processus phonologique ne s'applique qu'à des formes dérivées. On voit par cette simple définition que l'analyse d'une telle situation est problématique pour OT. En termes de dérivation traditionnelle, le NDEB se résume comme suit:

$\begin{array}{llll} & & \text { /YAZ/ } & \text { YBZ/ } \\ \text { 1. } & \mathrm{A} \rightarrow[\mathrm{B}] / \_\mathrm{Z} & \mathrm{YBZ} & - \\ \text { 2. } & {[\mathrm{B}] \rightarrow \mathrm{C} / \_\mathrm{Z}} & \mathrm{YCZ} & -\end{array}$

En (15), seul [B], ie. B dérivé se réalise en $\mathrm{C}$, tandis que /B/, ie. B lexical demeure inchangé.

Dans la distribution, souvent discutée, de certaines semi-voyelles en français standard, on observe l'alternance entre des formes comme [ви] (elle) roue et [виа : вwa] (elle) roua, la prononciation la plus courte [вша] étant la plus courante en style conversationnel. Cette alternance plaide en faveur d'un input /ьи $+\mathrm{a} /$ avec une voyelle pleine / $\mathrm{u} /$ en lexique et une règle de formation des semi-voyelles en grammaire. Il existe en effet un autre mot roi, qui n'est pas sujet à l'alternance et se prononce invariablement [вwa]; par optimisation du lexique, son input ne peut être que /ьwa/. La grammaire verra donc une contrainte pénalisant les séquences de voyelles hautes (pleines) dominer l'identité vocalique de ces mêmes voyelles. Par contre la marque sera elle même dominée par l'identité aux glides, pour assurer le contraste phonémique. 


$$
\begin{array}{lll}
\text { IDENT-Glide }= & \text { les glides d'input restent des glides en output } \\
* \mathrm{~V}(\mathrm{ht})+\mathrm{V}= & \text { pas de suites de voyelles hautes } \\
\mathrm{IDENT}-\mathrm{V}(\mathrm{ht})= & \text { une voyelle haute d'input reste une voyelle haute en output }
\end{array}
$$

Comme il est d'usage, la variation stylistique s'exprime par un réagencement des contraintes. La grammaire du style relâché aura l'ordre (16a), celles des locuteurs qui disent [вua] pour roua en style soigné aura l'ordre donné en (16b).

$$
\begin{aligned}
& \text { a. IDENT-Glide } \gg * V(h t)+V \gg \text { IDENT-V(ht) } \\
& \text { b. IDENT-Glide } \gg \text { IDENT-V(ht) } \gg * V(h t)+V
\end{aligned}
$$

Le défi pour OT provient du fait, encore une fois souvent discuté, que dans certains contextes la formation de glides est bloquée. Ainsi, à partir d'une racine /tьu/trou, on pourrait s'attendre à entendre une forme *[tьwa] pour troua, tout comme à partir d'une racine /ьu/ roue on a généré [вwa] pour roua. Il n'en est rien; [tвиа] est la seule prononciation possible. Si roua peut se prononcer en une seule syllable et que troua doit se prononcer en deux, c'est donc la forme de l'attaque qui fait la différence, d'où le filtre *OLGV proposé dès le début des années 1970:

*OLGV : " "pas de suite obstruante + liquide + glide + voyelle"

Or, il ne peut s'agir d'une contrainte de surface puisqu'il existe des formes comme [tbwa] trois, qui ne peuvent se prononcer que monosyllabiques. Ce qui doit être bloqué n'est pas une forme de surface, mais un processus; l'optimalité classique est ici démunie de moyens.

Ce phénomène a fait l'objet d'un grand nombre d'analyses, certaines structurales basées sur la bonne formation des constituants de la syllabe, d'autres purement morphologiques exploitant la présence de frontières de morphèmes dans certains mots, d'autres hybrides ou prosodiques. Ce cas de figure s'oppose exactement aux cas de "chain-shift", où seuls les éléments non-dérivés sont affectés par un processus. Dans ces deux cas, l'analyse OT peine, puisqu'il lui est impossible de distinguer entre formes lexicales et formes dérivées. Ici encore, il faut connaître à la fois le but à atteindre et le chemin à suivre. La grammaire doit engendrer la semi-voyelle tout en limitant la façon de l'obtenir: c'est bien un chemin qui s'avère impraticable. En langage ordinaire, on pourrait établir que OT-CC est l'antithèse du "n'importe comment, mais tout sauf ça" d'OT.

Il est pertinent de noter que OT-CC n'est pas le seul modèle qui s'efforce de retrouver une dimension de profondeur en réincorporant certains aspects de DT. C'est déjà la lignée dans laquelle s'inscrivaient des propositions plus anciennes, notamment le modèle OT-Stratal. Dans ce dernier modèle, on maintient les strates de la phonologie lexicale dérivationnelle (LP, d'où récemment LP-OT), dont l'existence avait été argumentée et motivée de façon convaincante. En OT-Stratal, l'ordre des contraintes change de strate à strate, les priorités du module lexical - ou plus généralement d'un module plus profond - n'étant pas les mêmes que celles du module post-lexical - ou d'un modèle plus superficiel. Autrement dit, en OT-Stratal, chaque strate contient sa propre grammaire. Mais à l'intérieur de chaque strate, l'évaluation continue à s'opérer en parallèle.

OT-Stratal constitue donc un modèle hybride, particulièrement pertinent lorsqu'on est confronté à des différences de priorité interstratales que l'on peut relier à des différences de domaines d'application ou de structures morphologiques. Mais lorsque les processus qui entrent en interaction appartiennent à la même composante morphologique et partagent un même domaine d'application, il devient spécieux de les séparer en des strates différentes, même si on aboutit à l'heureux résultat de faire disparaître l'opacité. Le procédé est artificiel: l'opacité ne disparaît que parce que l'interaction ne peut plus exister dans la même strate.

Si la morphologie n'est pas absente dans les deux exemples cités dans cette section (présence de frontières de morphèmes dans batterie et troua / roua, absence dans patrie et trois / roi), il reste démontré qu'il existe de nombreux cas d'opacité où la morphologie ne joue aucun rôle. Par exemple, Montreuil (sous 
presse) analyse un cas d'opacité multiple dans les racines simples du français régional de Lorraine et d'Alsace, où l'approche stratale se révèlerait particulièrement inadaptée.

\section{Conclusion}

Dans ce travail, plusieurs fragments phonologiques du français en style relâché ont été réanalysés à la lumière du modèle OT-CC. A la différence des modèles strataux qui utilisent une grammaire différente pour chaque strate, OT-CC évalue en une seule grammaire non pas des formes uniques, mais des chaînes de candidats qui relient l'input à l'output par une série de formes phonologiques qui diffèrent entre elles de façon minimale. Chaque forme - ou maillon - d'une chaîne diffère du maillon précédent par un écart de fidélité. Du maillon initial, soit la plus harmonique des interprétations fidèles de l'input, au maillon final, lorsque le point de convergence est atteint, la grammaire reste stable. Pour être bien formées, les chaînes doivent obéir à des critères d'harmonicité et de gradience.

L'esprit de DT perdure en filigrane. C'est non seulement le maillon final qui est évalué mais aussi la chaîne par laquelle la grammaire y aboutit. De cette façon, OT-CC nous oblige à repenser la nature de ce qu'une grammaire évalue: ce ne sont plus de simples formes phonétiques en sortie, mais plutôt des chemins phonologiques. Lorsque les séquences d'écarts mal formées (mal débutantes, non-harmoniques, non-gradientes...) sont pénalisées même si elles conduisent au bon maillon final, c'est tout l'ensemble du phénomène phonologique qui s'éclaire. Tout en gardant les avantages reconnus d'une évaluation en parallèle, la phonologie de l'optimalité se donne ainsi le moyen de retrouver la dimension de profondeur qui s'était trouvée perdue lors de l'abandon des dérivations.

Dans la mesure où OT est un modèle d'évaluation susceptible de s'appliquer à toutes les composantes linguistiques d'une langue - et même au-delà -, il est clair que cette démarche comporte en elle-même une dimension interdisciplinaire et suggère des possibilités d'application en dehors de la phonologie. Ce qui vaut des dérivations en phonologie vaut des transformations en syntaxe. Partout où l'approche reste générative, ie. dans toute entreprise linguistique où il y a des opérations de déplacement ou de mouvement, et où un output doit être engendré à partir d'un input de manière non-déclarative, la démarche qui est dessinée ici peut se révéler fructueuse. Pour atteindre le but, il faut éclairer le chemin.

\section{Références bibliographiques}

Carton, F et al. (1983). Les accents des français. Paris: Hachette.

Duez, D. (1995). On spontaneous French speech: aspects of the reduction and contextual assimilation of voiced stops. Journal of Phonetics, 23, 407-427.

Ernestus, M. (2000). Voice Assimilation and Segment Reduction in Casual Dutch, a corpus-based study of the phonology-phonetics interface. LOT: Utrecht.

Gow, D. W. (2002). Does English coronal place assimilation create lexical ambiguity? Journal of Experimental Psychology: Human Perception and Performance, 28, 163-179.

Gow, D. W., \& IM, A. M. (2004). A cross-linguistic examination of assimilation context effects. Journal of Memory and Language, 51, 279-296.

Hallé, P. A., Chéreau, Céline, \& Segui, Juan. (2000). Where is the /b/ in "absurde" [apsyrd]? It is in the French listeners' mind. Journal of Memory and Language, 43, 618-639.

Jesney, K. (2009). Positional Faithfulness, Non-locality and the Harmonic Serialism Solution. Rutgers Optimality Archives: ROA-1018.

Jun, J. (1995). Perceptual and Articulatory Factors in Place Assimilation: An optimality theoretic approach. Thèse de doctorat, UCLA, Los Angeles.

Kimper, W. (2008). Local optionality and Harmonic Serialism. Rutgers Optimality Archives: ROA-988.

Kiparsky, P. (1985). Some consequences of Lexical Phonology. Phonology Yearbook, 2, 85-138. 
Kuzla, C. (2003). Prosodically-conditioned variation in the realization of domain-final stops voicing assimilation of domain-initial fricatives in German. 15th International Congress of Phonetic Sciences, 2829-2832. Barcelona, Spain.

McCarthy, J. J. (2006). Slouching towards Optimality: Coda Reduction in OT-CC. Phonological Studies, 7.

McCarthy, J. J. (2007). Hidden Generalizations: Phonological Opacity in Optimality Theory. London: Equinox.

McCarthy, J. J. (2008). The gradual path to cluster simplification. Phonology 25, 2: 271-319

Mohanan, K. P. (1986). The Theory of Lexical Phonology. Studies in Natural Language and Linguistic Theory, 6. Dordrecht: D. Reidel Publications.

Montreuil, J.-P. (2009). Les sonantes palatales du bas-normand. Journées d'Etudes Linguistiques: JEL 2009. Nantes: LLING EA3827.

Montreuil, J.-P. (sous presse). Multiple opacity in Eastern Regional French. Linguistics Symposium on Romance Languages: LSRL-39. Amsterdam: John Benjamins.

Nolan, F. (1992). The descriptive role of segments: Evidence from assimilation. Laboratory phonology II: Gesture, segment, prosody, 261-280. Cambridge: Cambridge University Press.

Nolan, F., Holst, T., \& Kuhnert, B. (1996). Modeling [s] to []] accommodation in English. Journal of Phonetics, 24, 113-137.

Pruitt, K. (2008). Iterative foot optimization and locality in stress systems. Rutgers Optimality Archives: ROA-999

Quené, H., van Rossum, M. A., \& van Wijck, M. (1998). Assimilation and Anticipation in Word Perception. Fifth International Conference on Spoken Language Processing, Sydney, Australia.

Riggs, D. (2008). Opacity in Icelandic: Transparency and OT with candidate chains. Northeastern Linguistics Society: NELS. New York: Ithaca.

Snoeren, N. D. (2005). Variation phonologique en production et perception de la parole: le phénomène de l'assimilation. Paris: Thèse doctorale, Paris-5.

Snoeren, N. D., Hallé, P. A. \& Segui, J. (2006). A voice for the voiceless: Production and perception of assimilated stops in French. Journal of Phonetics, 34, 2: 241-268.

Snoeren, N. D., Segui, Juan \& Hallé, Pierre A. (2008). On the role of regular phonological variation in lexical access: Evidence from voice assimilation in French. Cognition, 108, 2: 512-521.

Walker, D. (1984). The Pronunciation of Canadian French. Ottawa: University of Ottawa Press.

Weber, A. (2001). Help or hindrance: How violation of different assimilation rules affects spoken-language processing. Language \& Speech, 44, 95-118.

Weber, A. (2002). Assimilation violation and spoken-language processing: A supplementary report. Language and Speech, 45 (2), 37-46.

Wetzels, W. L., \& Mascaró, J. (2001). The typology of voicing and devoicing. Language, 77, 207-244.

Wolff, M. (2008). Optimal Interleaving. University of Massachussetts at Amherst: PhD dissertation.

${ }^{1}$ Dans tous les exemples de cet article, je ne prends pas en compte la présence possible d'un e muet final (à quelque niveau d'analyse que ce soit). Les assimilations dont il va être question traitent de manière semblable les suites $\mathrm{C \# C}$ et les suites Cə\#C.

${ }^{2}$ En présence d'une plosive, l'assimilation de voix n'aboutit pas à une géminée, puisqu'il demeure une différence de tension ou de force (lenis vs. fortis); en ce sens, la transcription [pikdymidi] est plus correcte que ne le serait *[pigdymidi]. Lorsque cette assimilation se couple avec l'assimilation de hauteur, qui ne concerne que certaines fricatives, l'output est alors une vraie géminée, comme dans [ga $\left.\int \mathrm{o}\right]$.

${ }^{3}$ Dans cet article, la terminologie technique restera limitée, notamment dans la formulation des paraphrases. 
978-2-7598-0534-1, Paris, 2010, Institut de Linguistique Française

Conférence plénière

DOI $10.1051 / \mathrm{cmlf} / 2010266$

${ }^{4}$ Par souci de simplification, je laisse ici [ə] exprimer la position du schwa. On ne donnera pas à ce symbole son interprétation traditionnelle. La présence sous-jacente de schwa n'est pas mise en question, mais sa nature reste à définir. 